\title{
Analysis of Gene Effects Controlling Yield Contributing Traits in Grass pea (Lathyrus sativus L.)
}

\author{
Gonmei, R. ${ }^{2}$, Jeberson, M. S. ${ }^{1 *}$, Manish Kumar ${ }^{2}$, Singh, N. B. ${ }^{2}$, Shashidhar, K. S. ${ }^{1}$ and Ph. Ranjit Sharma ${ }^{2}$ \\ ${ }^{1}$ AICRP (MULLaRP), ${ }^{2}$ Dept. of Genetics \& Plant Breeding, Directorate of Research, CAU, Imphal, Manipur (795 004), India
}

\section{Corresponding Author}

Jeberson, M. S.

e-mail: samuel8142@gmail.com

\author{
Article History \\ Article ID: AR1898 \\ Received in 07 ${ }^{\text {th }}$ September, 2018 \\ Received in revised form $29^{\text {th }}$ October, 2018 \\ Accepted in final form $31^{\text {st }}$ October, 2018
}

\begin{abstract}
The seven parents and their $21 \mathrm{~F}_{1} \mathrm{~s}$ were grown in randomized block design with 3 replications during rabi 2015-16. In the field experiment, observations on 12 quantitative characters were recorded for genetic analysis. The analysis of variance for combining ability revealed significant differences among crosses for all the characters studied except for days to first flowering. In case of general combining ability effects, RLS 3004-5 and Pusa 24 were identified as the most promising parents for involving in hybridization programme. On the basis of specific combining ability effects, five crosses exhibited highly significant SCA effects for seed yield plant ${ }^{-1}$ with two to six other yield components. Among cross combinations, Prateek×RLS 3009-2, DLY 13-7×Pusa 24, DLY 13-7×RLS 3004-5 and RLS 3004-5×Bio L 212 were identified as the most promising crosses for improvement of seed yield per plant and three to six of its component traits. The manifestation of heterosis for seed yield was evidenced by superiority of hybrids over better parent and over standard check variety (Bio L 212). Out of 21 cross combinations, 5 crosses exhibited significant heterosis over their better parent as well as standard check. Overall on the basis of results of mean performance, including GCA and SCA effects and standard heterosis, Prateek $\times$ RLS 3009-2, DLY 13-7×Pusa 24 and DLY 137×RLS 3004-5 were identified as the most promising cross combinations for improvement of seed yield in grasspea.
\end{abstract}

Keywords: Grasspea, gene effects, yield contributing traits

\section{Introduction}

Lathyrus sativus L. (grass pea) is traditionally cultivated in India, Bangladesh and Ethiopia for its high degree of adaptability under adverse climatic conditions, low input requirement (Urga et al., 2005 and Kumari, 2001). Grasspea (Lathyrus sativus $L$.) has chromosome number $2 n=14$, belongs to the family Fabaceace, subfamily Papilionoideae, tribe Vicieae. Botanically it is a self-pollinated annual grain legume grown for its grain but also used for fodder or green manure (Rahman et al., 1995). It is rich in proteins (28.7-34.2\%) (Sammour, 2007) and possesses good qualities of essential amino-acids and micronutrients, thereby providing nutritional security to its consumers, who are mostly belonging to the poor section of the society. Though India is the world's largest producer of pulses, it imports a large amount of pulses to meet the growing domestic needs (Gowda, 2013). India grows a variety of pulse crops under a wide range of agro-climatic conditions and is the largest producer, consumer and processor of pulses in the world, contributing around $25-28 \%$ of the total production (Anon., 2011). The total pulse production of India in the year 2012-13 was 18.34 MT in contrast with 17.09 MT of year 2011-12. The production of grasspea in the year 2012-13 is 0.43 MT with the area coverage of 0.58 mha (Anon., 2013).
In the past, it had got more attention as a hardy pulse crop adapted to extreme environmental situations like drought and rain-fed, where soil quality was poor, excessive rainfall or flooding along with resistance to a range of damaging pests (Kaul et al., 1986; Palmer et al., 1989; Campbell et al., 1994). But, it harbors high neurotoxin ( $\beta$-N-oxalylamino alanine) content in seed and leaf (Grela et al., 2001) which warrants immediate effort for its elimination or reduction to improve nutritional quality. The presence of this toxin $\beta$ - ODAP has been the major constraint for promotion of this crop for commercial cultivation (Parihar et al. 2015). Therefore cultivation of low-ODAP toxin $(<0.1 \%) / O D A P$ free grasspea varieties or cultivars with higher yields having desirable attributes like disease and pest resistance along with matching production technologies are highly desirable. And there is no suitable and well adapted high yielding varieties of grasspea with low or free toxin available yet for the State of Manipur, we need to breed HYVs for the State. Here lies the importance of grasspea breeding programme with the main objectives to develop high yielding disease resistant varieties, better seed quality and wider adaptability. Hence the present investigation was carried out using half-diallel analysis with the following objectives: a. to study the general and specific combining ability (GCA and SCA) effects in Lathyrus; and b. to 
estimate the extent of heterosis in the inetrvarietal crosses of Lathyrus.

\section{Materials and Methods}

The present field investigation was conducted at the research field of the Department of Plant Breeding and Genetics, College of Agriculture, Central Agricultural University, Imphal during Rabi season during 2014-15 and 2015-16. The experimental field is situated at $24^{\circ} 51$ ' $\mathrm{N}$ latitude $93^{\circ} 56^{\prime} \mathrm{E}$ longitudes and at an altitude of 790 metre above msl. The climate of Imphal is subtropical with an average annual rainfall of about $1212 \mathrm{~mm}$ which is distributed mainly during the five monsoon months from June to October. The mean annual maximum and minimum temperature are $35{ }^{\circ} \mathrm{C}$ and $5{ }^{\circ} \mathrm{C}$ respectively. Humidity during the most part of the year range from $70-80 \%$ with an average annual sunshine of about 2231 hours. The soil of experimental site is clay in texture with acidic soil in reaction. It is high in organic carbon content, medium in available nitrogen content and with high in available potassium content. The $\mathrm{pH}$ ranges from 5.6-6.8. In the present investigation, seven (7) diverse lines of Lathyrus possessing different morphological and productive attributes were used (Table 1). The seeds were obtained from the AICRP (MULLaRP), IIPR, Kanpur, Uttarpradesh. The experiment took

\begin{tabular}{lcc}
\hline \multicolumn{3}{l}{ Table 1: Genotypes, pedigree and their sources } \\
\hline Genotypes & \multicolumn{1}{c}{ Pedigree } & \multicolumn{1}{c}{ Sources } \\
\hline 1. Bio L-212 & Somaclone of Pusa-24 & IARI, New delhi \\
2. DLY 13-7 & Pusa-24 X Ratan & Dholi, Bihar \\
3. Prateek & Somaclone of Pusa 24 & IGKV, Raipur \\
4. Pusa-24 & Selection from germplasm & IARI, New delhi \\
5. RLS 3004-5 & RW-12 X Ratan & IGKV, Raipur \\
6. RLS 3006-3 & Ratan X JRL-2 & IGKV, Raipur \\
7. RLS 3009-2 & Ratan X JRL-2 & IGKV, Raipur \\
\hline
\end{tabular}

two cropping seasons. In the first season of Rabi 2014-15, $\mathrm{F}_{1}$ hybrid seeds were produced following diallel cross analysis without reciprocals using the seven parental lines by hand emasculation and pollination technique and obtain seeds of $21 \mathrm{~F}_{1}$ crosses. In the next crop season of Rabi 2015-16, all the seeds of $21 \mathrm{~F}_{1}$ cross combination with the seven parental lines totally 28 treatments were grown in the RBD with three replications. Each parents and $F_{1} s$ in single row $4 \mathrm{~m}$ long were grown with the spacing of $30 \times 10 \mathrm{~cm}$ between and within rows, respectively. The observations were recorded on five randomly selected plants from parents and $F_{1} s$ in each replication. The observations were recorded, viz; days to first flowering, days to $50 \%$ flowering, days to maturity, plant height $(\mathrm{cm})$, number of pods plant ${ }^{-1}$, number of primary branches plant ${ }^{-1}$, pod length $(\mathrm{cm})$, number of seeds pod ${ }^{-1}$, seeds yield plant ${ }^{-1}(\mathrm{~g})$, biological yield plant ${ }^{-1}(\mathrm{~g}), 100$ seeds weight $(\mathrm{g})$ and harvest index ( $\mathrm{HI}) \%$. The data were analysed by method 2 model I of Griffing (1956).

\section{Results and Discussion}

The analysis of variance to test the differences between sets of progenies included in the study in a diallel set of 21 F1s (without reciprocals) and seven parents for the characters viz. days to first flowering, days to $50 \%$ flowering, days to maturity, plant height, number of pods plant ${ }^{-1}$, pod length, number of seeds pod $^{-1}$, seed yield plant ${ }^{-1}$, biological yield plant $^{-1}, 100$ seed weight and harvest index are presented in Table 2. The differences among genotypes were found to be highly significant for all the characters studied as shown by mean squares. This shows the sufficient variability available in the material studied.

The mean per se performance is considered as the prime criterion in evaluating the parents and the hybrids. The per se performance of the parents was good indicator for their GCA effects (Table 3). The parents RLS 3004-5 is good general combiner for seed yield, pod length, Prateek for plant height, number of pods plant ${ }^{-1}$ and number of seeds pod ${ }^{-1}$, Prateek and DLY-13-7 for good combiner for plant height. Pusa 24 for days to first flowering, 50\% flowering, days to maturity, 100 seeds weight and harvest index. Similar results were found by Singh et al. (2001) and Singh et al. (2010)b. The superior specific cross combinations for seed yield per plant viz., RLS 3004-5×Bio L 212 had positive GCA effects for one of their parents. This cross combination showed good mean performance for seed yield, pod length and biological yield plant $^{-1}$ respectively. These results corroborate the results obtained by Awasthi et al. (2001) and Singh et al. (2005).

\subsection{Analysis of variance for combining ability}

Analysis of variance for combining ability revealed that variance due to general combining ability (GCA) and specific combining ability (SCA) were highly significant for all the characters studied except for days to first flowering, indicating the importance of both the additive and non-additive genetic components of variation for this character (Table 4) Borah (2009). However, the estimates of SCA variance were higher than the GCA variance for most of the characters viz. plant height, No. pods plant ${ }^{-1}$, seed yield plant ${ }^{-1}$, number of seeds pod $^{-1}$, biological yield plant ${ }^{-1}, 100$ seed weight and harvest index $(\%)$, indicating the predominance of non-additive variance in controlling the expression of these characters. These findings are in conformity with the findings of and Singh et al. (2010)b and Zaman and Hazarika (2005).

\subsection{Analysis of general combining ability (GCA) effects}

The GCA effects of the parents are consolidated in Table 5 and Table 6. Estimation of GCA effects indicates that it was possible to pick up a good general combiners for all the characters because the combining ability of parents were consistent for all the yield components. The parent Pusa-24 recorded significantly negative GCA effects for $50 \%$ flowering, days to maturity and positively significant for number of 


\begin{tabular}{|c|c|c|c|c|c|c|c|}
\hline \multirow[t]{2}{*}{ Source of variation } & \multirow[t]{2}{*}{ d.f. } & \multicolumn{6}{|c|}{ Mean sum of squares } \\
\hline & & $\begin{array}{l}\text { Days to first } \\
\text { flowering }\end{array}$ & $\begin{array}{c}\text { Days to } 50 \% \\
\text { flowering }\end{array}$ & $\begin{array}{l}\text { Days to } \\
\text { maturity }\end{array}$ & $\begin{array}{l}\text { Plant height } \\
(\mathrm{cm})\end{array}$ & $\begin{array}{l}\text { No. of primary } \\
\text { branches plant }{ }^{-1}\end{array}$ & $\begin{array}{l}\text { No. of pods } \\
\text { plant }^{-1}\end{array}$ \\
\hline 1. Replication & 2 & 14.39 & $8.58^{* *}$ & $13.54^{* *}$ & 1.72 & $1.40^{* *}$ & $60.34^{*}$ \\
\hline 2. Treatment & 27 & $9.14^{*}$ & $4.73^{* *}$ & $7.28^{* *}$ & $111.43^{* *}$ & $2.63^{* *}$ & $173.00^{* *}$ \\
\hline 3. Parents & 6 & 4.41 & $5.21^{* *}$ & $4.30^{* *}$ & $148.59^{* *}$ & 0.18 & 32.83 \\
\hline 4. Hybrids & 20 & 8.75 & $3.25^{* *}$ & $7.14^{* *}$ & $93.52^{* *}$ & $3.01^{* *}$ & $204.70^{* *}$ \\
\hline 5. Parents Vs. Hybrids & 1 & $45.43^{* *}$ & $31.43^{* *}$ & $28.00^{* *}$ & $246.63^{* *}$ & $9.72^{* *}$ & $379.85^{* *}$ \\
\hline 6. Error & 54 & 5.28 & 0.99 & 1.02 & 5.31 & 0.15 & 14.76 \\
\hline \multirow[t]{2}{*}{ Source of variation } & d.f. & \multicolumn{6}{|c|}{ Mean sum of squares } \\
\hline & & $\begin{array}{l}\text { Pod length } \\
(\mathrm{cm})\end{array}$ & $\begin{array}{l}\text { No. of seeds } \\
\text { pod }^{-1}\end{array}$ & $\begin{array}{l}\text { seed yield } \\
\text { plant }^{-1}(\mathrm{~g})\end{array}$ & $\begin{array}{l}\text { biological yield } \\
\text { plant }^{-1}(\mathrm{~g})\end{array}$ & 100 seed wt. (g) & $\begin{array}{c}\text { Harvest index } \\
(\%)\end{array}$ \\
\hline 1. Replication & 2 & $0.07^{* *}$ & 0.16 & 2.65 & $9.72^{*}$ & 0.69 & 58.27 \\
\hline 2. Treatment & 27 & $0.10^{* *}$ & $0.22^{* *}$ & $11.70^{* *}$ & $47.39^{* *}$ & $4.09^{* *}$ & $183.70^{* *}$ \\
\hline 3. Parents & 6 & $0.19^{* *}$ & $0.23^{*}$ & $4.10^{* *}$ & $18.76^{* *}$ & $5.86^{* *}$ & 115.32 \\
\hline 4. Hybrids & 20 & $0.08^{* *}$ & $0.20^{* *}$ & $13.57^{* *}$ & $57.44^{* *}$ & $3.30^{* *}$ & $192.85^{* *}$ \\
\hline 5. Parents Vs. Hybrids & 1 & 0.02 & $0.52^{*}$ & $19.84^{* *}$ & $18.01^{* *}$ & $9.37^{* *}$ & $410.91^{* *}$ \\
\hline 6. Error & 54 & 0.01 & 0.08 & 0.95 & 1.95 & 0.30 & 54.75 \\
\hline
\end{tabular}

$*, * *$ significant at $(p=0.05)$ and $(p=0.01)$ levels respectively

Table 3: Mean per se performance of parents and crosses for days to first flowering, days to $50 \%$ flowering, days to maturity, plant height, No. of primary branches, No. of pods plant ${ }^{-1}$, pod length, No. of seeds pod ${ }^{-1}$, seed yield plant ${ }^{-1}$, biological yield plant $^{-1}, 100$ seed weight and harvest index in a half-diallel cross of grasspea

\begin{tabular}{|c|c|c|c|c|c|c|c|c|c|c|c|c|}
\hline Parent/Cross & DFF & DF & DM & $\mathrm{PH}$ & NPBP & NPP & PL & NSP & SYP & BYP & SW & $\mathrm{HI}$ \\
\hline Prateek×DLY-13-7 & 85.00 & 92.67 & 130.67 & 43.77 & 6.53 & 24.73 & 2.73 & 3.97 & 6.34 & 14.13 & 7.48 & 45.90 \\
\hline Prateek×Pusa 24 & 87.00 & 92.33 & 132.00 & 35.20 & 5.70 & 25.07 & 2.58 & 3.38 & 4.76 & 10.27 & 6.55 & 45.37 \\
\hline Prateek $\times$ RLS 3004-5 & 85.67 & 92.00 & 130.33 & 25.00 & 3.17 & 25.70 & 2.50 & 3.44 & 4.72 & 9.42 & 8.24 & 50.14 \\
\hline Prateek×Bio L- 212 & 86.67 & 92.33 & 130.33 & 37.67 & 3.87 & 27.00 & 2.28 & 3.50 & 6.53 & 10.97 & 6.68 & 59.59 \\
\hline Prateek $\times$ RLS 3009-2 & 86.33 & 91.67 & 130.76 & 41.27 & 4.43 & 37.43 & 2.64 & 3.89 & 12.43 & 22.55 & 8.80 & 55.52 \\
\hline Prateek×RLS 3006-3 & 85.00 & 91.67 & 130.67 & 33.40 & 3.47 & 26.20 & 2.45 & 3.27 & 6.05 & 11.03 & 7.80 & 56.49 \\
\hline DLY-13-7×Pusa 24 & 86.67 & 91.67 & 132.00 & 41.30 & 3.47 & 47.13 & 1.67 & 3.50 & 10.12 & 22.73 & 8.73 & 44.00 \\
\hline DLY-13-7×RLS 3004-5 & 88.33 & 94.00 & 133.67 & 36.47 & 4.27 & 40.20 & 2.63 & 3.53 & 9.53 & 17.68 & 7.39 & 53.83 \\
\hline DLY-13-7×Bio L- 212 & 87.00 & 92.33 & 130.33 & 40.53 & 6.10 & 38.60 & 2.61 & 3.40 & 7.34 & 12.62 & 7.45 & 58.08 \\
\hline DLY-13-7×RLS 3009-2 & 87.67 & 91.67 & 131.00 & 38.07 & 3.40 & 24.93 & 2.35 & 3.93 & 4.41 & 7.80 & 9.55 & 56.85 \\
\hline DLY-13-7×RLS 3006-3 & 86.67 & 91.67 & 131.67 & 30.40 & 3.43 & 26.67 & 2.28 & 3.17 & 6.25 & 10.14 & 7.47 & 61.56 \\
\hline Pusa $24 \times$ RLS 3004-5 & 85.00 & 91.33 & 129.33 & 34.75 & 3.50 & 27.64 & 2.42 & 3.63 & 5.93 & 10.80 & 6.90 & 55.78 \\
\hline Pusa 24×BioL-212 & 86.00 & 91.00 & 130.33 & 43.80 & 3.57 & 36.03 & 2.63 & 3.80 & 9.65 & 11.84 & 9.27 & 81.43 \\
\hline Pusa $24 \times$ RLS 3009-2 & 85.00 & 90.67 & 129.67 & 31.50 & 3.67 & 32.23 & 2.60 & 3.10 & 5.96 & 9.95 & 8.22 & 60.11 \\
\hline Pusa $24 \times$ RLS 3006-3 & 85.00 & 91.33 & 129.33 & 46.27 & 3.47 & 53.73 & 2.72 & 3.43 & 8.03 & 17.96 & 9.92 & 45.34 \\
\hline RLS 3004-5×Bio L-212 & 84.67 & 91.00 & 129.00 & 45.20 & 5.47 & 47.50 & 2.94 & 3.77 & 10.62 & 20.47 & 7.90 & 51.97 \\
\hline RLS 3004-5×RLS 3009-2 & 85.33 & 91.67 & 130.67 & 34.93 & 3.43 & 36.00 & 2.68 & 3.13 & 7.75 & 15.16 & 8.48 & 51.31 \\
\hline RLS $3004-5 \times R L S$ 3006-3 & 85.33 & 91.33 & 130.33 & 37.53 & 3.53 & 38.00 & 2.62 & 3.43 & 6.67 & 12.95 & 9.29 & 53.28 \\
\hline
\end{tabular}




\begin{tabular}{|c|c|c|c|c|c|c|c|c|c|c|c|c|}
\hline Parent/Cross & DFF & DF & DM & $\mathrm{PH}$ & NPBP & NPP & PL & NSP & SYP & BYP & SW & $\mathrm{HI}$ \\
\hline Bio L-212×RLS 3009-2 & 91.33 & 93.33 & 133.33 & 32.33 & 3.43 & 33.90 & 2.45 & 3.33 & 6.33 & 11.27 & 9.25 & 57.05 \\
\hline Bio L-212×RLS 3006-3 & 88.33 & 92.67 & 132.67 & 41.33 & 3.77 & 32.97 & 2.71 & 3.57 & 6.15 & 10.27 & 7.70 & 59.49 \\
\hline RLS 3009-2×RLS 3006-3 & 89.33 & 95.00 & 135.00 & 44.70 & 3.43 & 34.40 & 2.60 & 3.77 & 5.83 & 11.39 & 6.20 & 50.99 \\
\hline Prateek & 87.33 & 93.00 & 132.00 & 25.17 & 3.47 & 44.90 & 2.33 & 3.70 & 4.87 & 10.87 & 7.53 & 44.95 \\
\hline DLY-13-7 & 90.00 & 95.33 & 134.33 & 25.21 & 3.30 & 35.60 & 2.28 & 3.50 & 4.47 & 8.39 & 5.94 & 53.39 \\
\hline Pusa 24 & 87.00 & 91.33 & 130.33 & 44.17 & 3.23 & 40.57 & 2.75 & 3.27 & 6.67 & 11.83 & 8.87 & 56.34 \\
\hline RLS 3004-5 & 89.67 & 94.00 & 133.00 & 33.87 & 2.90 & 36.00 & 3.02 & 3.60 & 7.76 & 15.04 & 8.32 & 52.18 \\
\hline Bio L -212 & 88.67 & 93.00 & 132.67 & 33.03 & 3.10 & 40.67 & 2.63 & 3.13 & 5.60 & 15.49 & 7.54 & 39.13 \\
\hline RLS 3009-2 & 87.33 & 94.67 & 132.33 & 36.33 & 3.20 & 38.53 & 2.59 & 2.90 & 6.33 & 13.41 & 4.88 & 47.84 \\
\hline RLS 3006-3 & 87.67 & 93.00 & 132.33 & 39.67 & 3.67 & 36.80 & 2.58 & 3.27 & 6.90 & 11.29 & 7.93 & 55.11 \\
\hline $\mathrm{CV}$ & 2.6427 & 1.07 & 0.76 & 6.24 & 10.16 & 10.87 & 11.18 & 8.15 & 14.10 & 10.63 & 6.93 & 13.78 \\
\hline
\end{tabular}

DFF: Days to first flowering; DF: Days to 50\% flowering; DM: Days maturity; PH: Plant height $(\mathrm{cm})$; NPBP: No. of primary branches plant ${ }^{-1}$; NPP: Number of pods plant ${ }^{-1}$; PL: Pod length $(\mathrm{cm})$; NSP: Number of seeds pod ${ }^{-1}$; SYP: Seed yield plant ${ }^{-1}$ (g); BYP: Biological yield plant ${ }^{-1}$ (g); SW: 100 seed wt. (g); HI: Harvest index (\%)

\begin{tabular}{|c|c|c|c|c|c|c|c|}
\hline \multirow[t]{2}{*}{ Source of variation } & \multirow[t]{2}{*}{ d.f. } & \multicolumn{6}{|c|}{ Mean sum of squares } \\
\hline & & $\begin{array}{l}\text { Days to first } \\
\text { flowering }\end{array}$ & $\begin{array}{l}\text { Nodes to first } \\
\text { flowering }\end{array}$ & $\begin{array}{l}\text { Days to } 50 \% \\
\text { flowering }\end{array}$ & $\begin{array}{l}\text { Days to } \\
\text { maturity }\end{array}$ & $\begin{array}{l}\text { Plant height } \\
\text { (cm) }\end{array}$ & $\begin{array}{l}\text { No. of pods } \\
\text { plant }^{-1}\end{array}$ \\
\hline GCA & 6 & 3.00 & $2.13^{* *}$ & $2.63^{* *}$ & $43.16^{* *}$ & $0.71^{* *}$ & $27.76^{* *}$ \\
\hline SCA & 21 & 3.06 & $1.42^{* *}$ & $2.37^{* *}$ & $35.42^{* *}$ & $0.93^{* *}$ & $66.21^{* *}$ \\
\hline Error & 54 & 1.76 & 0.33 & 0.34 & 1.77 & 0.05 & 4.92 \\
\hline$s^{2} g$ & & 0.14 & 0.20 & 0.25 & 4.60 & 0.07 & 2.54 \\
\hline$s^{2} s$ & & 1.30 & 1.09 & 2.03 & 33.65 & 0.87 & 61.29 \\
\hline$s^{2} g / s^{2} s$ & & 0.11 & 0.18 & 0.12 & 0.14 & 0.08 & 0.04 \\
\hline \multirow[t]{2}{*}{ Source of variation } & d.f. & \multicolumn{6}{|c|}{ Mean sum of squares } \\
\hline & & $\begin{array}{l}\text { Pod length } \\
(\mathrm{cm})\end{array}$ & $\begin{array}{l}\text { No. of seeds } \\
\text { pod }^{-1}\end{array}$ & $\begin{array}{l}\text { seed yield } \\
\text { plant }^{-1}(\mathrm{~g})\end{array}$ & $\begin{array}{c}\text { biological yield } \\
\text { plant }^{-1}(\mathrm{~g})\end{array}$ & $\begin{array}{c}100 \text { seed } \\
\text { wt. }(\mathrm{g})\end{array}$ & $\begin{array}{l}\text { Harvest index } \\
(\%)\end{array}$ \\
\hline GCA & 6 & $0.06^{* *}$ & $0.06^{* *}$ & $1.42^{* *}$ & $4.80^{* *}$ & $0.85^{* *}$ & $25.11^{* *}$ \\
\hline SCA & 21 & $0.03^{* *}$ & $0.08^{* *}$ & $4.6^{* *}$ & $18.94^{* *}$ & $1.51^{* *}$ & $71.55^{* *}$ \\
\hline Error & 54 & 0.00 & 0.03 & 0.32 & 0.65 & 0.10 & 18.25 \\
\hline$s^{2} g$ & & 0.01 & 0.00 & 0.12 & 0.46 & 0.08 & 0.76 \\
\hline$s^{2} s$ & & 0.02 & 0.05 & 4.29 & 18.29 & 1.41 & 53.30 \\
\hline$s^{2} g / s^{2} s$ & & 0.29 & 0.06 & 0.03 & 0.02 & 0.06 & 0.01 \\
\hline
\end{tabular}

$*, * *$ significant at $(p=0.05)$ and $(p=0.01)$ levels respectively

pods plant ${ }^{-1}$ and 100 seed weight. The parent RLS 3004-5 was found to be good general combiner for dwarf plant height, pod length, seed yield plant ${ }^{-1}$, biological yield plant ${ }^{-1}$ and 100 seeds weight. The similar results were reposted by Kumar and Jain (2002). On the basis of overall performance across 12 characters, the RLS 3004-5 and Pusa 24 were identified as the most promising parents because both the parents were noticed either good or average general combiners for seed yield and other yield contributing characters. These parents may serve as valuable parents for hybridization programme or multiple crossing programme to achieve good segregants. These results were in confirmity with the results of Esposito et al. (2013), while they were studied with field pea.

\subsection{Analysis of specific combining ability (SCA) effects}

The magnitude of SCA effects is of prime importance in selecting the cross combinations with higher probability of obtaining transgressive segregates (Table 7 and 8). Out of 
Table 5: Estimates of General combining ability effects for days to first flowering, days to $50 \%$ flowering, days to maturity, plant height, No. of primary branches and No. of pods/plant in a half-diallel cross of grass pea

\begin{tabular}{lcccccc}
\hline Parents & $\begin{array}{c}\text { Days to first } \\
\text { flowering }\end{array}$ & $\begin{array}{c}\text { Days to 50\% } \\
\text { maturity }\end{array}$ & $\begin{array}{c}\text { Days to matu- } \\
\text { rity }\end{array}$ & $\begin{array}{c}\text { Plant height } \\
\text { (cm) }\end{array}$ & $\begin{array}{c}\text { No. of primary } \\
\text { branches }\end{array}$ & $\begin{array}{c}\text { No. of pods } \\
\text { per plant }\end{array}$ \\
\hline 1. Prateek & -0.60 & -0.07 & -0.31 & $-3.16^{*}$ & $0.36^{*}$ & $-2.96^{*}$ \\
2. DLY-13-7 & 0.62 & $0.59^{*}$ & $0.73^{*}$ & $-1.57^{*}$ & $0.33^{*}$ & -1.02 \\
3. Pusa 24 & -0.78 & $-0.93^{*}$ & $-0.90^{*}$ & $2.90^{*}$ & -0.11 & $2.26^{*}$ \\
4. RLS 3004-5 & -0.23 & 0.00 & -0.23 & $-1.50^{*}$ & $-0.19^{*}$ & 0.49 \\
5. BioL-212 & 0.62 & -0.00 & -0.01 & $1.31^{*}$ & $0.17^{*}$ & $1.64^{*}$ \\
6. RLS 3009-2 & 0.44 & $0.44^{*}$ & $0.40^{*}$ & 0.04 & $-0.29^{*}$ & -0.74 \\
7. RLS 3006-3 & -0.08 & 0.04 & 0.32 & $1.98^{*}$ & $-0.27^{*}$ & 0.33 \\
SEm \pm & 0.41 & 0.18 & 0.18 & 0.41 & 0.07 & 0.68 \\
CD $(p=0.05)$ & 0.82 & 0.35 & 0.36 & 0.82 & 0.14 & 1.37 \\
\hline
\end{tabular}

$*, * *$ significant at $(p=0.05)$ and $(p=0.01)$ levels respectively

Table 6: Estimates of General combining ability effects for pod length, number of seeds pod ${ }^{-1}$, seed yield plant ${ }^{-1}$, biological yield plant ${ }^{-1}, 100$ seed weight and harvest index in a half-diallel cross of grass pea

\begin{tabular}{lcccccc}
\hline Parents & Pod length $(\mathrm{cm})$ & $\begin{array}{c}\text { No. of seeds } \\
\text { pod }^{-1}\end{array}$ & $\begin{array}{c}\text { Seed yield } \\
\text { plant }^{-1}(\mathrm{~g})\end{array}$ & $\begin{array}{c}\text { Biological yield } \\
\text { plant }(\mathrm{g})\end{array}$ & $\begin{array}{c}100 \text { seed } \\
\text { weight }(\mathrm{g})\end{array}$ & $\begin{array}{c}\text { Harvest index } \\
(\%)\end{array}$ \\
\hline 1. Prateek & $-0.08^{*}$ & $0.12^{*}$ & $-0.54^{*}$ & $-0.55^{*}$ & $-0.26^{*}$ & $-2.95^{*}$ \\
2. DLY-13-7 & $-0.12^{*}$ & 0.08 & -0.28 & -0.35 & $-0.33^{*}$ & -0.27 \\
3. Pusa 24 & 0.03 & -0.05 & 0.26 & 0.24 & $0.49^{*}$ & 1.70 \\
4. RLS 3004-5 & $0.14^{*}$ & 0.04 & $0.59^{*}$ & $1.28^{*}$ & $0.21^{*}$ & -0.97 \\
5. BioL-212 & 0.03 & -0.02 & 0.26 & 0.37 & 0.04 & 1.83 \\
6. RLS 3009-2 & -0.01 & -0.09 & -0.01 & -0.01 & $-0.30^{*}$ & -0.21 \\
7. RLS 3006-3 & 0.00 & -0.07 & -0.30 & $-0.97^{*}$ & 0.14 & 0.88 \\
SEm \pm & 0.02 & 0.05 & 0.17 & 0.25 & 0.10 & 1.32 \\
CD $(p=0.05)$ & 0.04 & 0.10 & 0.35 & 0.50 & 0.19 & 2.64 \\
\hline
\end{tabular}

$*, * *$ significant at $(p=0.05)$ and $(p=0.01)$ levels respectively

five crosses, the cross combinations, Prateek $\times$ RLS 3009-2 and RLS 3004-5×Bio L-212 exhibited significant SCA effects for seed yield with seven other yield components. The cross Pusa 24×Bio L-212 exhibited significant SCA effects for seed yield along with other four yield components while the DLY13-7×Pusa 24 exhibited significant SCA effects for seed yield along with three other yield components. The cross DLY-137×RLS 3004-5 exhibited significant SCA effects for seed yield with other two yield components. Ranjan et al. (2005) and Zaman and Hazarika (2005) were also obtained similar results while studying combining ability in fieldpea.

In general, maximum crosses showing significant SCA effects, were invariably associated with better by performance for respective traits. The results were in agreement with those obtained by Bhardwaj and Kolhi (1998), Ranjan et al. (2005) and Singh et al. (2005) whom concluded that mean performance of the crosses was closely associated with SCA effects. Hence selection of the crosses on the basis of heterotic response should prove effective.

On the basis of both SCA effects and mean values, the cross, Prateek $\times$ RLS 3009-2 appeared to be the best combination for seed yield with days to $50 \%$ flowering, number of pods plant $^{-1}$, number of seeds pod ${ }^{-1}$, pod length, number of primary branches plant ${ }^{-1}, 100$ seeds weight and biological yield plant ${ }^{-1}$ followed by DLY-13-7× Pusa 24 for seed yield plant $^{-1}$ with number of pods plant ${ }^{-1}, 100$ seeds weight and biological yield plant $^{-1}$ and the cross RLS 3004-5×Bio L-212 with first flowering, $50 \%$ flowering, days to maturity, pod length, number of pods plant ${ }^{-1}$, number of primary branches plant ${ }^{-1}$ and biological yield plant $^{-1}$. Other researchers (Sofi et al. 2006 and Dhillon et al. 2007) also reported similar results.

From the Table 9, it is revealed that most of the good specific cross combinations for different characters involved parents 


\begin{tabular}{|c|c|c|c|c|c|c|}
\hline Crosses & $\begin{array}{l}\text { Days to first } \\
\text { flowering }\end{array}$ & $\begin{array}{l}\text { Days } 50 \% \\
\text { flowering }\end{array}$ & $\begin{array}{l}\text { Days to } \\
\text { maturity }\end{array}$ & $\begin{array}{l}\text { Plant height } \\
(\mathrm{cm})\end{array}$ & $\begin{array}{c}\text { No. of primary } \\
\text { branches plant }{ }^{-1}\end{array}$ & $\begin{array}{l}\text { No. of pods } \\
\text { plant }^{-1}\end{array}$ \\
\hline Prateek×DLY 13-7 & -1.99 & -0.27 & $-1.18^{*}$ & $11.61^{* *}$ & $1.99^{* *}$ & $-6.61^{* *}$ \\
\hline Prateek×Pusa- 24 & 1.42 & 0.92 & $1.78^{* *}$ & -1.42 & $1.59^{* *}$ & $-9.56^{* *}$ \\
\hline Prateek×RLS 3004-5 & -0.47 & -0.34 & -0.56 & $-7.23^{* *}$ & $-0.86^{* *}$ & $-7.15^{* *}$ \\
\hline Prateek×Bio L-212 & -0.32 & 0.06 & -0.78 & $2.63^{*}$ & $-0.52^{*}$ & $-6.99^{* *}$ \\
\hline Prateek×RLS 3009-2 & -0.47 & $-1.12^{*}$ & -0.85 & $7.50^{* *}$ & $0.51^{*}$ & $5.81^{* *}$ \\
\hline Prateek×RLS 3006-3 & -1.29 & -0.71 & -0.78 & $-2.31^{*}$ & $-0.48^{*}$ & $-6.49^{* *}$ \\
\hline DLY 13-7×Pusa 24 & -0.14 & -0.42 & 0.74 & $3.09^{*}$ & $-0.60^{*}$ & $10.56^{* *}$ \\
\hline DLY $13-7 \times R L S$ 3004-5 & 0.97 & 0.99 & $1.74^{* *}$ & $2.65^{*}$ & 0.27 & $5.40^{* *}$ \\
\hline DLY 13-7 X Bio L-212 & -1.21 & -0.60 & $-1.81^{* *}$ & $3.90^{* *}$ & $1.74^{* *}$ & 2.65 \\
\hline DLY 13-7×RLS 3009-2 & -0.36 & $-1.79^{* *}$ & $-1.56^{*}$ & $2.71^{*}$ & $-0.49^{*}$ & $-8.64^{* *}$ \\
\hline DLY 13-7×RLS 3006-3 & -0.84 & $-1.38^{*}$ & -0.81 & $-6.90^{* *}$ & $-0.48^{*}$ & $-7.97^{* *}$ \\
\hline Pusa $24 \times$ RLS $3004-5$ & -0.95 & -0.16 & -0.96 & $-3.53^{* *}$ & -0.06 & $-10.44^{* *}$ \\
\hline Pusa $24 \times$ BioL-212 & -0.81 & -0.42 & -0.18 & $2.70^{*}$ & -0.35 & -3.19 \\
\hline Pusa $24 \times$ RLS $3009-2$ & -1.62 & $-1.27^{*}$ & $-1.26^{*}$ & $-8.32^{* *}$ & 0.22 & $-4.62^{*}$ \\
\hline Pusa $24 \times$ RLS 3006-3 & -1.10 & -0.19 & $-1.52^{* *}$ & $4.50^{* *}$ & -0.01 & $15.82^{* *}$ \\
\hline RLS 3004-5×Bio L-212 & $-2.69^{*}$ & $-1.34^{*}$ & $-2.18^{* *}$ & $8.49^{* *}$ & $1.62^{* *}$ & $10.05^{* *}$ \\
\hline RLS 3004-5×RLS 3009-2 & -1.84 & $-1.19^{*}$ & -0.93 & -0.50 & 0.06 & 0.92 \\
\hline RLS 3004-5×RLS 3006-3 & -1.32 & $-1.12^{*}$ & $1.18^{* *}$ & -0.16 & 0.13 & 1.85 \\
\hline Bio L-212×RLS 3009-2 & $3.31^{* *}$ & 0.55 & $1.52^{* *}$ & $-5.91^{* *}$ & -0.30 & -2.32 \\
\hline Bio L-212×RLS 3006-3 & 0.82 & 0.29 & 0.93 & 1.15 & 0.01 & $-4.23^{*}$ \\
\hline RLS 3009-2×RLS 3006-3 & 2.01 & $2.10^{* *}$ & $2.85^{* *}$ & $5.79^{* *}$ & 0.14 & -0.52 \\
\hline $\mathrm{SEm} \pm(\mathrm{Sij})$ & 1.191 & 0.516 & 0.523 & 1.194 & 0.203 & 1.991 \\
\hline$C D(p=0.05)$ & 2.388 & 1.034 & 1.048 & 2.394 & 0.407 & 3.992 \\
\hline
\end{tabular}

$*, * *$ significant at $(p=0.05)$ and $(p=0.01)$ levels respectively

Table 8: Estimates of Specific combining ability effects for pod length, number of seeds pod ${ }^{-1}$, seed yield plant ${ }^{-1}$, biological yield plant $^{-1}, 100$ seed weight and harvest index

\begin{tabular}{|c|c|c|c|c|c|c|}
\hline Crosses & $\begin{array}{l}\text { Pod length } \\
(\mathrm{cm})\end{array}$ & $\begin{array}{l}\text { No. of seeds } \\
\text { pod }^{-1}\end{array}$ & $\begin{array}{l}\text { Seed yield } \\
\text { plant }^{-1}(\mathrm{~g})\end{array}$ & $\begin{array}{c}\text { Biological yield } \\
\text { plant }^{-1}(\mathrm{~g})\end{array}$ & $\begin{array}{l}100 \text { seed } \\
\text { weight }(\mathrm{g})\end{array}$ & $\begin{array}{c}\text { Harvest index } \\
(\%)\end{array}$ \\
\hline Prateek×DLY 13-7 & $0.36^{* *}$ & $0.30^{*}$ & 0.23 & $1.90^{*}$ & 0.20 & -4.56 \\
\hline Prateek×Pusa- 24 & 0.07 & -0.16 & $-1.89^{* *}$ & $-2.55^{* *}$ & -1.55 & -7.06 \\
\hline Prateek×RLS 3004-5 & $-0.13^{*}$ & -0.19 & $-2.26^{* *}$ & $-4.44^{* *}$ & 0.42 & 0.38 \\
\hline Prateek×Bio L-212 & $-0.25^{* *}$ & -0.07 & -0.12 & $-1.99^{* *}$ & $-0.97^{* *}$ & 7.00 \\
\hline Prateek×RLS 3009-2 & $0.15^{*}$ & $0.40^{* *}$ & $6.05^{* *}$ & $9.98^{* *}$ & 1.49 & 5.00 \\
\hline Prateek×RLS 3006-3 & -0.04 & -0.25 & -0.05 & -0.58 & 0.05 & 4.87 \\
\hline DLY 13-7×Pusa 24 & $-0.14^{*}$ & -0.01 & $3.20^{* *}$ & $9.71^{* *}$ & $0.71^{*}$ & $-11.10^{* *}$ \\
\hline DLY $13-7 \times R L S 3004-5$ & 0.04 & -0.08 & $2.28^{* *}$ & $3.63^{* *}$ & -0.36 & 1.39 \\
\hline DLY 13-7×Bio L-212 & $0.12^{*}$ & -0.13 & 0.42 & -0.53 & -0.132 & 2.84 \\
\hline DLY 13-7×RLS 3009-2 & -0.09 & $0.47^{* *}$ & $-2.23^{* *}$ & $-4.98^{* *}$ & $2.31^{* *}$ & 3.65 \\
\hline
\end{tabular}




\begin{tabular}{|c|c|c|c|c|c|c|}
\hline Crosses & $\begin{array}{l}\text { Pod length } \\
(\mathrm{cm})\end{array}$ & $\begin{array}{l}\text { No. of seeds } \\
\text { pod }^{-1}\end{array}$ & $\begin{array}{l}\text { Seed yield } \\
\text { plant }^{-1}(\mathrm{~g})\end{array}$ & $\begin{array}{l}\text { Biological yield } \\
\text { plant }^{-1}(\mathrm{~g})\end{array}$ & $\begin{array}{l}100 \text { seed } \\
\text { weight }(\mathrm{g})\end{array}$ & $\begin{array}{c}\text { Harvest index } \\
(\%)\end{array}$ \\
\hline DLY 13-7×RLS 3006-3 & $-0.17^{* *}$ & $-0.32^{*}$ & -0.11 & $-1.67^{* *}$ & -0.21 & 7.27 \\
\hline Pusa $24 \times$ RLS 3004-5 & $-0.32^{* *}$ & 0.17 & $-1.85^{*}$ & $-3.85^{* *}$ & -1.66 & 1.38 \\
\hline Pusa 24×BioL-212 & -0.01 & $0.39^{*}$ & $2.19^{* *}$ & $-1.90^{* *}$ & $0.87^{* *}$ & $24.23^{* *}$ \\
\hline Pusa $24 \times$ RLS 3009-2 & 0.01 & -0.23 & $-1.23^{*}$ & $-3.41^{* *}$ & 0.16 & 4.95 \\
\hline Pusa $24 \times$ RLS 3006-3 & $0.13^{*}$ & 0.08 & $1.14^{*}$ & $5.56^{* *}$ & 1.42 & $-10.92^{* *}$ \\
\hline RLS 3004-5×Bio L-212 & $0.19^{* *}$ & 0.27 & $2.84^{* *}$ & $5.68^{* *}$ & -0.23 & -2.56 \\
\hline RLS $3004-5 \times R L S 3009-2$ & -0.02 & -0.29 & 0.24 & 0.76 & $0.70^{*}$ & -1.18 \\
\hline RLS 3004-5×RLS 3006-3 & -0.09 & -0.01 & -0.56 & -0.48 & $1.07^{* *}$ & -0.30 \\
\hline Bio L-212×RLS 3009-2 & $-0.15^{* *}$ & -0.03 & -0.86 & $-2.22^{* *}$ & $1.64^{* *}$ & 1.76 \\
\hline Bio L-212×RLS 3006-3 & 0.10 & 0.18 & -0.75 & $-2.26^{* *}$ & -0.35 & 3.10 \\
\hline RLS 3009-2×RLS 3006-3 & 0.03 & $0.46^{* *}$ & -0.80 & -0.76 & -1.51 & -3.35 \\
\hline $\mathrm{SEm} \pm(\mathrm{Sij})$ & 0.05 & 0.15 & -0.51 & 0.72 & 0.28 & 3.83 \\
\hline $\mathrm{CD}(p=0.05)$ & 0.10 & 0.29 & 1.01 & 1.45 & 0.57 & 7.69 \\
\hline
\end{tabular}

$*, * *$ significant at $(p=0.05)$ and $(p=0.01)$ levels respectively

of low $\times$ low, low $\times$ average, averagexaverage, average $\times$ high and high $\times$ high general combining ability. However in majority of cases, the crosses exhibiting high SCA effects were found to have either or both of the parents as good general combiner

\begin{tabular}{|c|c|c|c|}
\hline Character & Sca effects & Gca effects & Per se performance \\
\hline Days to first flowering & RLS 3004-5×Bio L 212(-2.69) & $L \times A$ & 84.67 \\
\hline Days to $50 \%$ flowering & DLY 13-7×RLS 3009-2 (-1.79) & $\mathrm{H} \times \mathrm{H}$ & 91.67 \\
\hline \multirow[t]{2}{*}{ Days to maturity } & RLS 3004-5×Bio L 212(-2.18) & $L \times L$ & 129.00 \\
\hline & DLY 13-7×Bio L 212 (-1.81) & $H \times L$ & 131.00 \\
\hline \multirow[t]{2}{*}{ Plant height } & DLY 13-7×RLS 3006-3 (-6.89) & $\mathrm{L} \times \mathrm{H}$ & 30.40 \\
\hline & Bio L 212×RLS 3009-2 (-5.90) & $\mathrm{H} \times \mathrm{A}$ & 32.33 \\
\hline \multirow[t]{2}{*}{ No. of primary branches plant ${ }^{-1}$} & Prateek×DLY 13-7 (1.99) & $\mathrm{H} \times \mathrm{H}$ & 6.53 \\
\hline & DLY 13-7×BioL 212(1.74) & $\mathrm{H} \times \mathrm{H}$ & 6.10 \\
\hline \multirow[t]{2}{*}{ No. of pods plant ${ }^{-1}$} & Pusa 24×RLS 3006-3 (15.82) & $\mathrm{H} \times \mathrm{A}$ & 53.73 \\
\hline & DLY 13-7×Pusa 24 (10.56) & $\mathrm{L} \times \mathrm{H}$ & 47.13 \\
\hline Pod length & Prateek×DLY 13-7 (0.36) & $L \times L$ & 2.73 \\
\hline No. of seeds pod ${ }^{-1}$ & DLY 13-7×RLS 3009-2(0.47) & $A \times L$ & 3.93 \\
\hline \multirow[t]{5}{*}{ Seed yield plant ${ }^{-1}$} & Prateek×RLS 3009-2 (6.05) & $L \times L$ & 12.43 \\
\hline & DLY13-7×Pusa 24 (3.20) & $L \times A$ & 10.12 \\
\hline & RLS 3004-5×Bio L 212 (2.84) & $\mathrm{H} \times \mathrm{A}$ & 10.62 \\
\hline & DLY 13-7×Bio L 212(2.28) & LXA & 7.34 \\
\hline & Pusa 24×Bio L 212 (2.19) & $A \times A$ & 9.64 \\
\hline \multirow[t]{2}{*}{ Biological yield plant ${ }^{-1}$} & Prateek RLS 3009-2 (9.98) & $L \times L$ & 22.55 \\
\hline & DLY 13-7×Pusa 24 (9.71) & $L \times A$ & 22.73 \\
\hline \multirow[t]{2}{*}{100 seed weight } & DLY 13-7×RLS 3009-2 (2.31) & $L \times L$ & 9.55 \\
\hline & Bio L 212×RLS 3009-2 (1.64) & $A \times L$ & 9.25 \\
\hline Harvest index & Pusa 24×Bio L 212 (24.23) & $A \times A$ & 81.43 \\
\hline
\end{tabular}


for the character under reference. Kumar et al. (2006) and Patil and Navale (2006) reported that most of the promising cross is the one that involves parents with high GCA and shows high SCA effects. The major part of such variance would be fixable in later generations. Such crosses were Pusa $24 \times$ RLS $3009-2$ for $50 \%$ flowering, days to maturity, Pusa $24 \times R$ LS $3006-3$ for days to maturity, pods per plant,

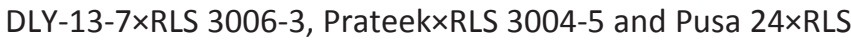
3004-5 for plant height, Prateek $\times$ DLY-13-7 for number of primary branches plant ${ }^{-1}$ and seeds pod ${ }^{-1}$, Prateek $\times$ Pusa 24 for number of primary branches per pod, Prateek $\times$ RLS 3009 2 for seeds per pod, DLY-13-7×Pusa 24 for pods plant ${ }^{-1}$ and 100 seeds weight, DLY-13-7×RLS 3004- 5 for seed yield plant ${ }^{-1}$ and biological yield per plan,DLY-13-7×Bio L 212 for number of primary branches plant ${ }^{-1}$, Pusa $24 \times$ Bio L 212 for 100 seeds weight, RLS 3004-5×RLS 3009-2 and RLS 3004-5× RLS 3006-3 for 100 seeds weight and RLS 3004-5×Bio L 212 for seed yield plant $^{-1}$, biological yield plant ${ }^{-1}$, pod length, number of primary branches plant ${ }^{-1}$ and number of pods plant ${ }^{-1}$. Recombination breeding through multiple crosses involving these hybrids would be desirable to breed genotypes combining these characters. The present findings are in tune with Singh et al. 2005, Singh et al., 2010a and Brar et al. (2012).

\section{Conclusion}

The overall picture from combining ability analysis revealed the predominant role of non-additive genetic variance for yield and yield components. On the basis general combining ability effects RLS 3004-5 and Pusa 24 were identified as most promising parents for involving in hybridization programme for generating desirable segregants. Overall on the basis of results of mean performance, SCA effects and standard heterosis, the cross combination Prateek $\times$ RLS 3009-2, DLY 13-7×Pusa 24 and DLY 13-7×RLS 3004-5 were identified as the most promising cross combinations to give transgressive segregants in later generations.

\section{References}

Anonymous, 2011. IIPR Vision 2030. Printed and published by the Director, Indian Institute of Pulses Research (ICAR), Kanpur-208024.

Anonymous, 2013. State of Indian Agriculture 2012-13. Printed and published by Directorate of Economics and Statistics, Ministry of Agriculture, Government of India. New Delhi.

Awasthi, S., Lavanya, R.G., Jain, R., 2009. Heterosis estimates of garden pea crosses. Trends in Biosciences 2(2), 45-47.

Borah, H.K., 2009. Studies on combining ability and heterosis in field pea (Pisum sativum L.). Legume Research 32(4), 255-259.

Brar, P.S., Dhall, R.K., Dinesh., 2012. Heterosis and combining ability in garden pea for yield and its contributing traits. Vegetable Science 39(1), 51-54.

Campbell C.G., Mehra, R.B., Agrawal, S.K., Chen, Y.Z., Abd El
Moneim, A.M., Khawaja, H.I.T., 1994. Current status and future strategy in breeding grasspea (Lathyrus sativus L.). Euphytica 73, 167-175.

Dhillon, T.S., Singh, M., Brar, P.S., 2007. Assessment of combining ability for some quantitative characters in pea. Crop improvement 34(1), 106-109.

Esposito, M. A., Gatti, H., Cravero, V.P., Anodo, F.S.L., Cointry, E.L., 2013. Combining ability and heterotic groups in Pea. Australian Journal of Crop Science 11, 1634-1641.

Gowda, C.L.L., Srinivasan, S., Gaur, P.M., Saxena, K.B., 2013. Enhancing the productivity and production of pulses in India, 145-159 (In) Climate Change and Sustainable Food Security. Shetty, P.K., Ayyappan, S., Swaminathan, M.S. (Eds.), National Institute of Advanced Studies, Bengalaru and Indian Council of Agricultural Research, New Delhi.

Grela, E.R., Studzinski, T., Matras, J., 2001. Antinutritional factors in seeds of Lathyrus sativus cultivated in Poland. Lathyrus Lathyrism Newsletter 2, 101-104.

Griffing, B., 1956. Concept of general and specific combining ability in relation to diallel crossing system. Australian Journal of Biological Science 9, 463-493.

Kaul, A.K., Islam, M.Q., Hamid, A., 1986. Screening of Lathyrus germplasm of Bangladesh for BOAA content and some agronomic characters. In Lathyrus and lathyrism. Kaul, A.K., Combs, D. (Eds.), 93-97., Third World Medical Research Foundation, New York, NY, USA.

Kumar, A., Jain, B.P., 2002. Combining ability status in pea. Indian Journal of Horticulture 59(2), 181-184.

Kumari, V., 2001. Field evaluation of grasspea (Lathyrus sativus L.) germplasm for its toxicity in the North-Western hills of India. Lathyrus Lathyrism Newsletter 2, 82-84.

Palmer, V.S., Kaul, A.K., Spencer, P.S., 1989. International network for the improvement of Lathyrus sativus and eradicators of lathyrism (INILSEL): ATWMRF initiative. In The grasspea, threat and promise (ed. P. S. Spencer), 219-223. Third world medical research foundation, New York, NY, USA.

Pandey, P.K., Singh, K.P., Kar, R.M., 1996. Combining ability analysis for some quantitative characters in garden pea. Annals of Agricultural Research 17(3), 230-234.

Parihar, A.K., Dixit, G.P., Singh, D., 2015. Gene interactions and genetics for yield and its attributes in grass pea (Lathyrus sativus L.) Journal of Genetics, DOI: 10.1007/ s12041-016-0722-2.

Patil, H.E., Navale, P.A., 2006. Combining ability in cowpea. Legume Research 29(4), 270-273.

Ranjan, S., Kumar, M., Pandey, S.S., 2005. Diallel analysis for yield and yield contributing characters in pea. Legume Research 38(3), 223-225.

Sammour R., Mustafa A.E.Z., Badr S., Tahr W. 2007. Genetic variations in accessions of Lathyrus sativus L., Acta Bot. Croat. 66 (1), 1-13, 2007.

Singh, H., Singh, M., Brar, P.S., 2005. Heterosis and combining ability studies for economic traits in genetically 
diverse lines of garden pea (Pisum sativum L.). Crop Improvement 32(1), 78-85.

Singh, I., Sandhu, J.S., Singh, J., 2010a. Combining ability for yield and its component in fieldpea. Journal of Food Legumes 23(2), 143-145.

Singh, K.P., Singh, H.C., Verma, M.C., 2010b. Genetic analysis for yield and yield traits in pea. Journal of Food Legumes, 23(2), 113-116.

Singh, K.P., Singh, H.C., Verma, M.C., 2010b. Genetic analysis for yield and yield traits in pea. Journal of Food Legumes, 23(2), 113-116.

Singh, N.K., Kumar, D., Kumar, N., Sing, D.N., 2001.
Combining ability for yied and its components in pea. Annals of Agricultural 22(4), 570-575.

Sofi, P., Rather, A.G., Wani, S.A., 2006. Combining ability and gene action studies over environments in field pea. Pakistan Journal of Biological Science 9(14), 2689-2692.

Urga, K., Fufa, H., Biratu, E., Husain, A., 2005. Evaluation of Lathyrus sativus cultivated in Ethiopia for proximate composition, minerals, ODAP and antinutritional components. African Journal of Food, Agriculture and Nutritional Development (AJFAND) 5(1), 1-15.

Zaman, S., Hazarika, G.N., 2005. Combining ability in pea. Legume Research 25(2), 105-108. 